\title{
MIXSELs - a new class of ultrafast semiconductor lasers
}

\section{Doctoral Thesis}

Author(s):

Maas, Deran

Publication date:

2008

Permanent link:

https://doi.org/10.3929/ethz-a-005761961

Rights / license:

In Copyright - Non-Commercial Use Permitted 


\title{
MIXSELS - A NEW CLASS OF ULTRAFAST SEMICONDUCTOR LASERS
}

\author{
A dissertation submitted to \\ E T H Z U R C H \\ for the degree of \\ D OCTOR OF SCIENCES \\ presented by \\ D ER A N J.H.C. M A A S \\ M.Sc. in Electrical Engineering, Eindhoven University of Technology \\ born on May 25, 1979 \\ citizen of the Netherlands \\ accepted on the recommendation of \\ Prof. Dr. U. Keller, Supervisor \\ Dr. T. Südmeyer, Co-Examiner \\ Prof. Dr. J. Faist, Co-Examiner
}

December 2008 


\section{Abstract}

Picosecond and femtosecond laser oscillators have enabled many breakthroughs in both fundamental science and industrial applications. However, so far these ultrafast lasers have not achieved the impact of continuous-wave lasers, which are used in various everyday life applications such as compact disk players, optical communication links or laser printers. One reason for the low market penetration is the complexity and cost of these sources. Currently, there are no suitable ultrafast laser sources for industrial high volume applications such as the optical clocking of microprocessors at gigahertz frequencies. Compact and reliable ultrafast lasers have also large potential in areas as diverse as biology, medicine, or metrology, which currently rely on bulky and complex ultrafast lasers such as titanium sapphire oscillators. In contrast to these laser systems, semiconductor lasers are ideally suited for mass production and allow a high level of integration which results in compact and simple devices.

In this thesis, we developed a novel type of ultrafast semiconductor laser. Our approach is based on the integration of a vertical external cavity surface emitting laser (VECSEL) and a semiconductor saturable absorber mirror (SESAM) into a single semiconductor structure. We refer to this class of devices as modelocked integrated external-cavity surface emitting lasers (MIXSEL).

The optically pumped VECSEL has received widespread interest in the past few years due to its capability of producing high average output powers in a diffractionlimited beam. The laser beam propagates vertically (perpendicularly) through the epitaxial layers. The total thickness of the epitaxial layers is small compared to the beam diameter of the pump laser allowing for very efficient heat removal. This makes the device power-scalable, i.e. the output power can be scaled upwards by increasing the pumped area, while the temperature difference in the semiconductor structure remains unchanged. Continuous wave output powers of up to $20 \mathrm{~W}$ in a diffraction limited beam have been obtained. Pulsed operation is obtained by modelocking with a SESAM inside the cavity.

The quantum well (QW) SESAMs used previously have a saturation energy comparable to that of the gain structure itself. Simulations and experiments show 
that stable modelocking is only obtained if the absorber saturates stronger than the gain. This has been realized by designing a cavity mode with a very small spot size on the SESAM compared to the gain structure. This unfortunately makes integration impossible.

A key challenge of this work was the development of saturable absorbers with saturation properties that enable modelocking with similar mode sizes in the gain and absorber layers. Semiconductor saturable absorber mirrors using quantum dot (QD) absorbers exhibit a larger freedom of design than standard QW absorbers. The dot density as an additional parameter in combination with the field enhancement allows for an independent control of saturation fluence and modulation depth. I present the first detailed study on the influence of QD growth parameters and post growth annealing on the macroscopic optical SESAM properties. The nonlinear reflectivity and the recombination dynamics were evaluated for a large set of QDSESAMs. We experimentally demonstrated for the first time that the dot density allows to precisely adjust the modulation depth at constant saturation fluence. Moreover, we showed that post growth annealing can reduce the saturation fluence to values below $10 \mu \mathrm{J} / \mathrm{cm}^{2}$. By modelocking a VECSEL with a QD-SESAM (i.e. using a folded cavity) we obtained more than $100 \mathrm{~mW}$ of average power at $50 \mathrm{GHz}$ pulse repetition rate using the same spot size on gain structure and absorber.

Optimization of the QDs required precise optical SESAM characterization. We developed a new method to perform wide dynamic range nonlinear reflectivity measurements. The measurement of the reflectivity has a very high accuracy of better than $0.05 \%$ for pulse energies over a dynamic range of more than four orders of magnitude. Even with this high accuracy, the setup is straightforward and has lower demands on the electronic equipment compared to previous methods.

Using optimized QD-layers, we demonstrated the first VECSEL with integrated saturable absorber. Our first MIXSEL generates $40 \mathrm{~mW}$ of average output power in 32-ps pulses at a repetition rate of $2.8 \mathrm{GHz}$. Improved cooling has increased the output power to $185 \mathrm{~mW}$. Advanced heat management will result in opticallypumped MIXSELs with multi-Watt average output powers similar to our previous modelocked VECSEL results with an external SESAM.

The final step towards even more compact and inexpensive ultrafast semiconductor lasers is electrical pumping. We developed design guidelines for electrically pumped VECSELs and MIXSELs. The realization of such devices will fill a gap in the performance spectrum of today's laser technology. 


\section{Kurzfassung}

Laseroszillatoren, die Piko- oder Femtosekundenpulse erzeugen, waren die Grundlage einer Vielzahl von wissenschaftlichen und industriellen Durchbrüchen. Dennoch haben Ultrakurzpuls-Laser bislang eine weitaus geringere Verbreitung als Laser im Dauerstrichbetrieb, welche in verschiedensten alltäglichen Anwendungen wie CD-Spielern, bei der optischen Nachrichtenübertragung oder in Laserdruckern genutzt werden. Gründe hierfür sind die hohe Komplexität und die hohen Kosten der momentan verfügbaren Ultrakurzpuls-Laserquellen. So gibt es beispielsweise derzeit keine serienmäßig herstellbaren Kurzpuls-Laser, die für die optische Takterzeugung von Mikroprozessoren bei Frequenzen im Gigahertzbereich geeignet wären. Kompakte und zuverlässige Kurzzeit-Laser haben ebenfalls ein grosses Potential in Bereichen wie Biologie, Medizin oder Metrologie, welche momentan noch auf unhandliche und komplexe Laserquellen, wie beispielsweise Titan-SaphirOszillatoren, angewiesen sind. Im Gegensatz zu diesen Lasersystemen eignen sich Halbleiterlaser ideal für die Massenproduktion, da durch weitgehende Integration kompakte und einfache Bauteile möglich sind.

Im Rahmen dieser Arbeit wurde ein neuer Typ von Kurzzeit-Halbleiterlasern entwickelt. Der Ansatz basiert dabei auf der Integration eines optisch gepumpten oberflächenemittierenden Halbleiterlasers mit externer Kavität (vertical external cavity surface emitting laser, VECSEL) und eines sättigbaren HalbleiterAbsorberspiegels (semiconductor saturable absorber mirror, SESAM) in eine einzige Halbleiterstruktur. Diese Kategorie von Lasern wird modengekoppelter integrierter oberflächenemittierender Halbleiterlaser mit externer Kavität (modelocked integrated external-cavity emitting laser, MIXSEL) genannt.

Der VECSEL hat, aufgrund seiner Eigenschaft hohe durchschnittliche Ausgangsleistung in einem beugungslimitierten Strahl zu erzeugen, in den letzten Jahren stark an Bedeutung gewonnen. Da der Laserstrahl vertikal (senkrecht) in den epitaktischen Schichten propagiert, wird eine effiziente Wärmeabfuhr durch die dünne Schichtstruktur hindurch gewährleistet. Dies wiederum ermöglicht die 
Skalierung der Ausgangsleistung des Bauteils, das heisst die Ausgangsleistung kann durch die Vergrösserung der gepumpten Fläche erhöht werden, während die Temperaturdifferenz im Halbleiter unverändert bleibt. Im Dauerstrichbetrieb wurde mit diesem Konzept in einem beugungslimitierten Strahl bisher eine Ausgangsleistung von $20 \mathrm{~W}$ erzeugt. Durch Modenkoppeln mit einem SESAM innerhalb des Resonators wird ein gepulster Betrieb erreicht.

Früher verwendete SESAMs mit Quantentopfabsorbern (quantum well, QW) haben eine Sättigungsenergie vergleichbar mit derjenigen der Verstärkerstruktur. Simulationen und Experimente zeigen allerdings, dass stabiles Modenkoppeln nur dann realisierbar ist, wenn der Absorber stärker sättigt als der Verstärker. Durch den Bau von Resonatoren mit sehr kleiner Modenfläche auf dem SESAM im Vergleich zum Verstärker kann dies zwar umgesetzt werden, Integration ist dadurch jedoch ausgeschlossen.

Eine der Hauptaufgaben dieser Arbeit war die Entwicklung eines sättigbaren Absorbers mit entsprechenden Sättigungseigenschaften, der Modenkoppeln bei gleicher Modengrösse sowohl in Verstärker- als auch in Absorberschichten ermöglicht. Im Gegensatz zu QW Absorbern bieten SESAMs mit Quantenpunktabsorbern (quantum dot, QD) die Möglichkeit einer unabhängigen Anpassung der Parameter. So ermöglicht die Punktdichte als zusätzlicher Parameter zusammen mit der Felderhöhung eine unabhängige Kontrolle von Sättingungsfluenz und Modulationstiefe. Im Rahmen dieser Arbeit wird die erste detaillierte Studie vorgelegt, in welcher der Einfluss von QD Wachstumsparametern und des thermischen Ausheilens nach dem Wachstum auf makroskopisch-optische SESAM Eigenschaften untersucht wurde. Für eine grosse Zahl von QD-SESAMs wurde die nichtlineare Reflexion und Rekombinationsdynamik gemessen, wodurch zum ersten Mal experimentell nachgewiesen werden konnte, dass die Punktdichte die genaue Einstellung der Modulationstiefe ermöglicht, während die Sättigungsfluenz konstant bleibt. Darüber hinaus wurde gezeigt, dass durch das thermische Ausheilen nach dem Wachstum die Sättigungsfluenz auf Werte unter $10 \mu \mathrm{J} / \mathrm{cm}^{2}$ reduziert werden kann. Modenkopplung eines VECSELs mit einem QD-SESAM, das heisst unter Verwendung eines gefalteten Resonators, erlaubte es, mehr als $100 \mathrm{~mW}$ mittlere Ausgangsleistung bei einer Repetitionsrate von $50 \mathrm{GHz}$ zu erreichen. Hierbei hatten die Modenflächen auf der Verstärkerstruktur und dem Absorber die gleiche Grösse. 
Eine QD Optimierung erfordert eine sehr genaue SESAM Charakterisierung. Zu diesem Zweck wurde ein neues Verfahren zur nichtlinearen Reflexionsmessung mit einem grossen Dynamikbereich entwickelt. Dieses weist mit einer absoluten Abweichung kleiner als $0.05 \%$ eine hohe Genauigkeit für Pulsenergien über einen Dynamikbereich von mehr als vier Zehnerpotenzen auf. Trotz seiner hohen Genauigkeit ist der Messaufbau unkompliziert und stellt im Vergleich zu früheren Messmethoden geringere Ansprüche an die elektronische Ausrüstung.

Unter Verwendung optimierter QD Schichten wurde das erste VECSEL mit integriertem, sättigbarem Absorber, das MIXSEL, entwickelt und demonstriert. Dieses erzeugt eine durchschnittliche Ausgangsleistung von $40 \mathrm{~mW}$ mit Pulsen von 32 ps bei einer Pulsrepetitionsrate von 2.8 GHz. Durch eine verbesserte Kühlung des Elements wurde die Ausgangsleistung auf $185 \mathrm{~mW}$ gesteigert. Eine Verbesserung des Wärmeabflusses im Halbleiter wird zu optisch gepumpten MIXSELn mit höherer durchschnittlicher Ausgangsleistung führen, die vergleichbar mit der von modengekoppelten VECSELn mit externen SESAMs sein wird.

Die letzte Stufe hin $\mathrm{zu}$ kleineren und kostengünstigeren KurzzeitHalbleiterlasern ist das elektrische Pumpen. Es wurden bereits Richtlinien für den Entwurf von elektrisch gepumpten VECSELn und MIXSELn entwickelt, deren Umsetzung eine Lücke im Spektrum der heutigen Lasertechnologie schliessen wird. 